\title{
The outcome of surgical resection in complete paraplegia patient due to thoracic schwannoma
}

\author{
Maria Monica' \\ Bair Ginting ${ }^{2}$
}

'General Physician, Bendan Regional Public

Hospital, Pekalongan, Indonesia

${ }^{2}$ Department of Neurosurgery, Bendan

Regional Public Hospital, Pekalongan, Indonesia
Cite this article:

Monica M, Ginting B. The outcome of surgical resection in complete paraplegia patient due to thoracic schwannoma. Neurologico Spinale Medico Chirurgico. 202I.4(2).78-83. DOI: I0.36444/nsmc.v4i2.I 55

\section{Corresponding author:}

Maria Monica

Bendan Regional Public Hospital

Binagriya Street B-IV No. 195, Medono,

Pekalongan - 5I III, Indonesia

monica_eagan@yahoo.co.id

\begin{abstract}
Spinal schwannoma is the most common benign intradural spinal tumor. However, studies regarding the neurological outcome of tumor resection in complete paraplegia patients are still lacking. The authors report a case of complete paraplegia due to primary intradural extramedullary low-grade schwannoma of the spinal cord. Surgery was performed 5 weeks after the paraplegia complaint first occurred. The patient did not regain motor nor sensory function in the 1-month follow-up, but resolution of pain was achieved. The patient was able to perform daily activity more comfortably. In the event of complete paraplegia, surgery should still be offered promptly as it can still benefit the patient. Possible factors that could have influenced the prognosis in our study are discussed.
\end{abstract}

Keywords: Spinal schwannoma, Spinal tumor, Paraplegia, Complete paralysis

\section{Introduction}

Accounting for almost $25 \%$ of all intradural spinal tumors, spinal schwannoma is the most common benign intradural tumor. The typical clinical manifestation is localized pain, radicular pain, and myelopathy, while symptoms of motor weakness usually occur at a later stage. ${ }^{1,2}$ The mainstay of treatment for spinal schwannoma is surgical resection, whether it is done through minimally invasive procedure or conventional one. While radiosurgery is reserved for selected cases (i.e., tumor recurrence, incomplete resection, or surgery not feasible due to tumor location and other comorbidities). ${ }^{3,4}$ The goal of surgical intervention is to do maximum possible resection to decompress the spinal cord, without worsening the pre-operative neurological status and maintaining the spinal stability. ${ }^{5}$ Complete resection can be achieved in $85 \%-97 \%$ of primary operated cases with more than 60 percent of patients experiencing improvement in neurological outcome..$^{4,6}$

To the best of the authors' knowledge, studies regarding the neurological outcome of surgical intervention in complete paraplegia due to spinal schwannoma are still limited. Most of the existing studies only discuss the surgical outcome of paraplegic patients due to spinal metastases. Li et al., found $66.7 \%$ of 135 patients with paraplegia due to metastasis, experienced improvement in neurologic status after surgery. ${ }^{7}$ While Yu Fan et al., found improvement of at least 1-Frankel grade in 37 patients (86\%) with preoperative Frankel B status who were operated before 72 -hours. ${ }^{8}$ 
Regarding study specific for spinal schwannoma, most of the included patients were not paraplegic. In a study by M. Seppälä et al., involving 187 spinal schwannoma patients, there were 53 patients who were unable to walk before surgery and only two of them were complete paraplegic. Of these 53 patients, 43 (81\%) were able to walk again including one patient who was paraplegic.9 Whereas in the study by Tarlov et al., four patients who were paraplegic due to spinal schwannoma experienced improvements in motor strength to the point that they were able to walk on their own.10 Nevertheless, because the two study above did not specifically discuss the outcome of only paraplegia cases, other neurological outcomes was not further differentiated. Thus, we aim to discuss the neurological outcome of surgical intervention particularly in complete paraplegia patient due to spinal schwannoma based on the current study and review of the literature

\section{Case Report}

\section{Presentation and Examination}

A 57-year-old woman came to the clinic with complaints of inability to walk for one week. The patient had a history of progressive back pain for one year, radiating pain, lower extremity numbness and weakness for 11 months, 10 months, and 9 months before coming to the clinic, respectively. The complaints were felt to be getting worse to the point that the patient was unable to move both of her lower extremities at all and could not feel starting from the lower abdomen to both feet. The pain was also bothersome, to the point of interfering with the patient daily activity (VAS 6). Occasional difficulty in defecation was also found without any complaint of micturition.

Neurological examination showed zero motoric strength (complete paraplegia) and hyporeflexia of both lower extremities, along with complete anesthesia below the level of T10 (Frankel A). Magnetic resonance imaging (MRI) revealed a strongly homogenous enhancement of extramedullary intradural solid mass compressing the spinal cord, measuring $3.44 \times 0.91 \times 1.2 \mathrm{~cm}$ at the level of T9-10. The mass was seen as hypointense on T1W1, T2W1 view, and hyperintense on the T2-STIR view. After the examination, surgery was immediately offered to the patient, but the patient refused initially. The patient finally agreed upon surgery after four weeks of initial presentation to the clinic, which is approximately five weeks since the complete paraplegia complaint and one year after the first initial complaint.
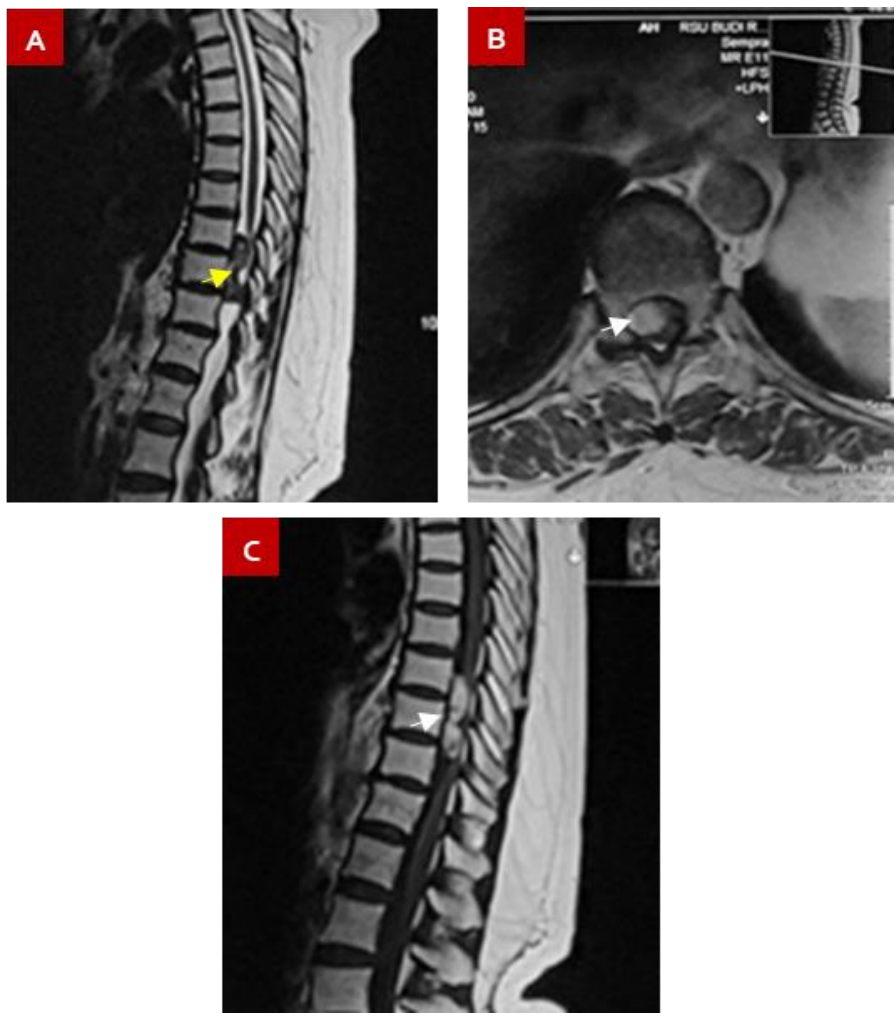

Figure 1. (A) Sagittal T2-weighted, (B) axial T1-weighted gadoliniumenhanced, and (C) sagittal T1-weighted gadolinium-enhanced views of preoperative magnetic resonance imaging. Image $A$ demonstrates a hypointense mass (yellow arrow) compressing the spinal cord. Image $B$ and $C$ show a T9-10 intradural extramedullary tumor with strong homogenous enhancement (white arrow).

\section{Operative procedure}

The patient underwent bilateral total laminectomy in T8-11 vertebrae and gross total tumor removal. The tumor was found to be reddish, soft in consistency, attached to the spinal nerve root, slightly attached to the spinal cord, and unattached to the duramater. Because total laminectomy was done at 3 spinal levels (T8-9, T9-10, and T1011) and more than $50 \%$ of facetectomy was performed, we decided to install pedicle screws to maintain spinal stability. Pedicle screw fixation was performed in the level of T8 and T11 bilaterally.

\section{Post-operative course}

The surgery and the post-operative course were uneventful. Immediately after the surgery, the patient noted a marked reduction of lower back pain and radiating pain. Before discharge, the pain was said to be very minimal. The author also noted an increase of physiological reflex on both lower extremities to a positive one. At one month of follow-up, complete paraplegia and complete anesthesia below the level of the T10 dermatome were found to be status quo as before surgery (Frankel A). But resolution of both types of pain was achieved 
and although the patient was wheelchair-bound, she was able to perform daily activity more comfortably. Occasional difficulty on defecation was also noted.
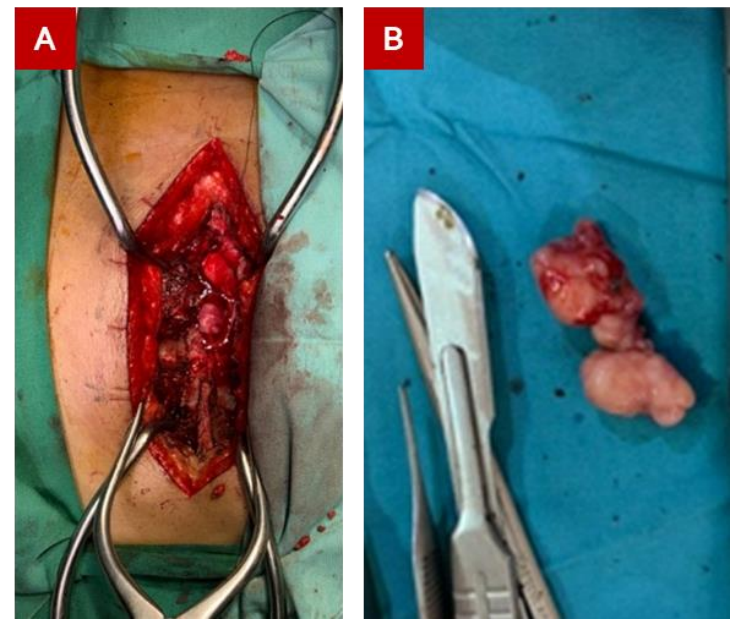

Figure 2. (A) Intraoperative photo showing the tumor (white arrow) encompassing the posterior surface of the spinal cord (grey arrow). Note that the duramater was retracted (arrowhead). (B) Gross pathology of the tumor.

\section{Histopathological examination}

The resected tumor was a discrete white and soft tissue measuring 4 x $1.5 \times 1 \mathrm{~cm}$ in dimension. Microscopically, the tumor was composed of round, oval, and spindle hyperchromatic to fine chromatin cells. The hypercellular areas formed the Antoni A component, partly with a whorl pattern, and some nuclei were arranged in palisades, forming Verocay Bodies. The hypocellular areas formed the Antoni B component. There were no signs of mitosis nor malignancy. The histopathological examination was consistent with conventional Schwannoma (WHO grade I).

\section{Discussion}

Spinal schwannoma produces a variety of symptoms depending on the tumor location. Local back pain and radiating pain are the most common presenting symptoms. The pain arises due to direct or indirect irritation of the nerve root or root compression by the tumor which causes disturbance of nerve conductivity. In the long term, growth in tumor size causes impairment of spinal column integrity and compression of the spinal cord, leading to progressively worsening myelopathy. Furthermore, the enlarged tumor also causes compression of the arteries, resulting in infarction of the nerve elements. Thus, motor deficits usually occur in the later stage. ${ }^{1,2}$ Complete paraplegia in acute condition may present due to direct nerve impairment, intra-tumoral bleeding, and microscopical enlargement that causes the tumor and its edema to compress the whole spinal canal, nerve root, and blood supply. ${ }^{10}$ In our study, complete paraplegia occurred progressively so the pathophysiology is more suitably explained by the mechanism described earlier.

Surgical intervention is the main treatment modality in an intradural extramedullary tumor, including spinal schwannoma. A maximal resection without worsening the preoperative neurological status and preserving the spinal stability is the gold standard for schwannoma surgery. ${ }^{5}$ Directly repairing the existing nerve tissue impairment is an impossible task. However, surgical therapy can provide an opportunity for the nerve to heal by relieving compression of the nerve tissue, arteries, and reducing edema that occurs.

The reversibility of the nerve damage that occurs could not be determined before surgery. Tarlov et al., recommended early resection of the lesion that compressed the spinal cord in the hope of improving its function. ${ }^{10}$ In a study by Jeon et al., $95 \%$ of the population study experienced symptoms improvement after surgery (median follow-up 15.9 months). ${ }^{2}$ While according to M. Seppälä et al., $31 \%$ of the population study still had complaints of paraparesis after surgery (median follow-up 12.9 months). ${ }^{9}$

Current surgical techniques for intradural tumor is generally divided into minimally invasive surgery (MIS) and Non-MIS, which both include hemilaminectomy or laminectomy, with or without pedicle screw fixation, use of microscope, and neurophysiological monitoring (NPHm). ${ }^{11}$ There is an increasing trend of using MIS and NPHm. Visually, MIS provides a clearer operative view and thus allow gentler operation maneuver to increase rate of total resection and reduce damage into the spinal cord and nerve root, intraoperative blood loss, and postoperative complication. ${ }^{5}$ However due to narrow surgical corridor, there is a significant learning curve in identifying the spinal anatomy. MIS is relatively contraindicated in large tumor (i.e., involving $\geq 2$ levels or $>16 \mathrm{~mm}$ axial diameter), extensive extraforaminal tumor, and hemorrhagic tumor. ${ }^{12}$ Meanwhile, NPHm is used to identify the degree of nerve damage and determine the functional role of a nerve root. The advantages of using IOM are still being debated. Some researchers argue that surgery without the use of $\mathrm{NPhM}$ could significantly cause more neurologic deficits. In contrast to this, others suggest that the use of IOM is not related with occurrence of new neurologic deficits and extent of tumor resection. ${ }^{1,3}$ In a developing country like Indonesia, MIS and NPHm are considered rare resources. We elected to not do MIS and NPHm because the patient will need to be transported far away, costing more money, and delaying the surgery even more. 
The most common surgical approach performed is total laminectomy via a standard posterior midline incision. ${ }^{3,13}$ This approach could cause damage to musculoligament structures and posterior bony elements, resulting in spinal instability and kyphosis. ${ }^{13}$ In addition, long incisions and excessive muscle dissection result in ischemic necrosis, massive hemorrhage, epidural fibrosis, and postoperative dorsal pain. ${ }^{5,11}$ Some surgeons prefer hemilaminectomy approach to preserve the paravertebral ligaments and muscles on the opposite side thereby reducing damage to the posterior structures of the vertebral column. The disadvantages of hemilaminectomy include the narrow operating field, which can increase the risk of nerve damage. ${ }^{5,13}$ Either hemilaminectomy or laminectomy did not cause a significant difference in spinal stability and gave comparable clinical outcomes. ${ }^{5}$ To date no clear guideline is available in selecting the surgical technique. Decision on which surgical technique will be performed and amount of bone exposed are based on tumor location, extent of extradural component, and surgeon preference. ${ }^{12,13}$ Because the tumor in our study was extending in two spinal levels (T9-T10), we elected to do laminectomy to achieve maximal tumor resection.

Instability is defined as the loss of the ability of the spine under physiological loads to maintain the connection between the vertebrae so that there is no damage or irritation to the spinal cord or nerve roots and no development of deformity with excessive pain. ${ }^{14}$ The incidence of spinal instability after laminectomy is $20 \%$ in adults and up to $45 \%$ in children. A biomechanical study by Nong et al., found that total laminectomy causes instability through increased lamina strain, increased transverse displacement, decreased rigidity, and decreased load-bearing capacity. The number of excised laminae is negatively correlated with the load-bearing capacity of the spine. ${ }^{15}$ Decreased strength and stability of the thoracic spine was observed after a two level laminectomy. ${ }^{16}$

The degree of kyphotic angulation that occurs after laminectomy is related to extent of facet joint removed. ${ }^{2}$ More than $50 \%$ of facetectomy will cause a decrease in the strength of the remaining joints so that fractures can occur even with physiological loads. ${ }^{14}$ Bilateral facetectomy at one or more levels can result in acute angular kyphosis which then cause spinal cord compression. ${ }^{2}$

The use of internal fixation is important in preventing spinal deformity and instability after laminectomy. ${ }^{2}$ Fixation with a pedicle screw can restore centrum strain, shift, rigidity, and load-bearing capacity back to $4 \%-11 \%$ of normal biomechanics. Therefore, pedicle screw can provide immediate stable fixation, control segmental movement in three dimensions, reduce clinical symptoms, and accelerate spinal function recovery. Several disadvantages in the use of internal fixation are longer hospital stay, higher infection rate, and increase risk of spinal degeneration in long-term. ${ }^{11,15}$ In this study, we chose to do internal fixation with pedicle screw because the patient was in risk of developing spinal instability and angular kyphosis due to the extent of total laminectomy (3 spinal levels i.e., T8-9, T9-10, and T1011) and facetectomy performed (more than $50 \%$ of facets were removed).

Degree of resection is divided by gross-total resection (GTR; i.e. no evidence of residual tumor was found on postoperative MRI), subtotal resection (STR; i.e. more than $90 \%$ resection was performed based on postoperative MRI), and partial resection (i.e. less than $90 \%$ resection was performed based on postoperative MRI. ${ }^{6,17}$ GTR should be attempted in all patients because the extent of resection correlates with the rate of tumor recurrence. ${ }^{3,6}$ Regardless, GTR can only be performed in $85 \%-97 \%$ cases of schwannoma. ${ }^{1,6}$ Total resection of spinal schwannoma is sometimes not possible because schwannoma consists of neoplastic Schwann cells which are nerve wraps and thus forcing excision of the tumor can cause injury to the wrapped root. ${ }^{1}$ Other condition that may complicate the resection are adhesions to the spinal cord due to bleeding, inflammation, or sub-pial localization; and the presence of other important structures that attach directly to extradural components, such as the vertebral arteries. ${ }^{2}$ In our study, we were able to completely removed the tumor. Though, no postoperative MRI was done due to financial issue.

The author did not find any specific study reference about the neurological outcome of surgery in complete paraplegia patients due to spinal schwannoma. Available articles were mostly discussing acute complete paraplegia; thus, they are not appropriate for this study. Li et $a l$. , described the American Spinal Injury Association Impairment Scale (AIS) outcome of surgical intervention in complete paraplegia patients due to spinal tumors. According to the study, 90 of 135 patients $(66.7 \%)$ had improved neurological status with 52 patients (38.5\%) having good improvement (AIS stage D and E) and 83 patients (61.5\%) having poor improvement (AIS stage B, C, and A). ${ }^{7}$

Some of the factors that are thought to influence the outcome of post-operative neurological status are the duration of the first symptoms to the paraplegia complaint, duration of the paraplegia to surgical intervention, and the preoperative neurological status. The longer the duration of the paraplegia and the shorter the duration between complete paraplegia to surgery, the better the patient's outcome after the surgery. ${ }^{1,7,9,10}$ Previous studies showed surgery within 48 hours of the paraplegia complaint could lead to a better outcome. Whereas in 
patients who passed this time window, surgery within one week was found to give a better outcome than after. Surgery performed more than 3 weeks after the onset of paraplegia had a 4.2-fold risk of having a worse outcome than surgery within 1 week of paralysis. ${ }^{7}$ This is due to the progressivity of the neurovascular damage around the spinal cord. Delay in surgery could cause the damage to be irreversible. ${ }^{1}$

In our study, complete resolution of pain was achieved but no improvement in motor or sensory deficits was found. Still, the surgery benefited the patient because currently, she was able to perform daily activity more comfortably. Perhaps delay in surgery and time interval between the first complaint to complete paralysis were major factors in the outcome of our study. The time interval between the first chief complaint (lower back pain) to complete paralysis was one year. Moreover, the patient had delayed the surgery for five weeks since the complete paralysis complaint. Thus, missing the window period mentioned above.

Other factors that need to be considered in the post-operative neurological outcome were the patient's general condition, the degree of tumor removal, possible trauma to the spinal cord during surgery, the treatment given to the paralyzed muscle, the level of patient cooperation, and muscle tone. ${ }^{7,10}$ Patients with spastic paralysis are theorized to have a better surgical outcome. Several possible explanations are central and peripheral sprouting, remyelination, muscle hypertrophy, pre-and post-synaptic upregulation, reorganization of the central nervous system, and lack of integrity of lower motor neurons (LMN). Damage to the LMN cell body was thought to not undergo any repair and cause permanent damage. ${ }^{7}$

In this present study, the patient had an upper motor neuron (UMN) lesion. Despite having a favorable prognostic factor, no motor improvement was observed. This indicated the duration between the first symptoms of paralysis symptoms and the duration of paralysis to surgery was a factor that had more influence on the outcome of the surgery. In addition, age could influence the outcome. Recuperation is harder in an older patient due to different neuronal activity and other comorbids. $^{7}$

Spinal schwannoma recurrence rate was found to be $1.9 \%$ $5.8 \% .{ }^{6}$ Significant factors contributing to recurrence rate were extent of resection and tumor size. Fehlings et al., found for every $1 \mathrm{~cm}$ increase in tumor size (especially cranial-caudal diameter), the risk of recurrence increased by 1.15 times. Patients with involvement of more than 1 spinal level had a $22.8 \%$ increased chance of recurrence. ${ }^{17}$ Whilst presence of neurofibroma mutation, tumor pathology, tumor location, use of postoperative radiotherapy, use of NPHm, and surgery technique was not significantly correlated with tumor recurrence. Nonetheless, the above factors may contribute to the extent of resection that can be performed. ${ }^{3,6,17}$ In our study, the tumor size was not considered large enough to be at risk of recur (less than $6.97 \pm 4.66 \mathrm{~cm}$ ) ${ }^{17}$ and complete resection was able to be performed. Therefore, we think that the recurrence rate of our patient's spinal schwannoma is low. Even if the tumor recurs in the same segment, the laminectomy performed will allow more space so that the posterior part of the vertebrae does not compress the spinal cord and nerve roots. The installation of pedicle screws will also allow the bone segments to fused so the tumor will not cause spinal instability.

The main limitation of this study is the short follow-up time, thus the final neurological outcome may not have been seen yet. The patient could still have an improvement in the long-term follow-up. Tarlov et al., showed average time needed for complete paraplegia patients to walk again was between 25 days - 14 months. ${ }^{10} \mathrm{~A}$ pathology-specific, prospective, larger-sample, and longer follow-up time studies are needed to provide better data to guide clinical decision making.

\section{Conclusion}

Surgical intervention should still be offered as soon as possible as it could still benefit the patient, even in the case of a long period of paraplegia. Although the patient in our study did not regain motor nor sensory function, resolution of pain had increased her tolerability in performing daily activities. Several factors that could affect the patient prognosis in our study were delay in surgery, the short time interval between the first complaint to complete paralysis, and patient age. A long post-operative follow-up is needed to determine the patients' final post-operative neurological status.

\section{Acknowledgment}

No Acknowledgment

\section{References}

1. Hohenberger C, Hinterleitner J, Schmidt N-O, et al. Neurological outcome after resection of spinal schwannoma. Clin Neurol Neurosurg. 2020;198:106127. DOI: 10.1016/j.clineuro.2020.106127

2. Jeon JH, Hwang HS, Jeong JH, et al. Spinal schwannoma; analysis of 40 cases. J Korean Neurosurg Soc. 2008;43(3):135-138. DOI: 


\subsection{0/jkns.2008.43.3.135}

3. Lenzi J, Anichini G, Landi A, et al. Spinal nerves schwannomas: Experience on 367 cases-historic overview on how clinical, radiological, and surgical practices have changed over a course of 60 years. Neurol Res Int. 2017;2017:3568359. DOI: $10.1155 / 2017 / 3568359$

4. Kufeld M, Wowra B, Muacevic A, et al. Radiosurgery of spinal meningiomas and schwannomas. Technol Cancer Res Treat. 2012;11(1):27-34. DOI: 10.7785/tcrt.2012.500231

5. Zong S, Zeng G, Du L, et al. Treatment results in the different surgery of intradural extramedullary tumor of 122 cases. PLoS One. 2014;9(11):e111495. DOI: 10.1371/journal.pone.0111495

6. Safaee M, Parsa AT, Barbaro NM, et al. Association of tumor location, extent of resection, and neurofibromatosis status with clinical outcomes for 221 spinal nerve sheath tumors. Neurosurg Focus FOC. 2015;39(2):E5. DOI: 10.3171/2015.5.focus 15183

7. Li S, Zhong $\mathrm{N}, \mathrm{Xu} \mathrm{W}$, et al. The impact of surgical timing on neurological outcomes and survival in patients with complete paralysis caused by spinal tumours. Bone Joint J. 2019;101B(7):872-879. DOI: 10.1302/0301-620X.101B7.BJJ-20181173.R1

8. Fan Y, Zhou X, Wang H, et al. The timing of surgical intervention in the treatment of complete motor paralysis in patients with spinal metastasis. Eur Spine J. 2016;25(12):4060-4066. DOI: $10.1007 / \mathrm{s} 00586-016-4406-7$

9. Seppälä MT, Haltia MJJ, Sankila RJ, et al. Long-term outcome after removal of spinal schwannoma: A clinicopathological study of 187 cases. $\quad J \quad$ Neurosurg. 1995;83(4):621-626. DOI:10.3171/jns.1995.83.4.0621

10. Tarlov IM, Herz E. Spinal cord compression studies. IV. Outlook with complete paralysis in man. AMA Arch Neurol Psychiatry. 1954;72(1):43-59.
DOI:10.1001/archneurpsyc.1954.02330010045003

11. Zong S, Zeng G, Xiong C, et al. Treatment results in the differential surgery of intradural extramedullary schwannoma of 110 cases. PLoS One. 2013;8(5):e63867. DOI: 10.1371/journal.pone.0063867

12. Lee SE, Jahng T-A, Kim HJ. Different surgical approaches for spinal schwannoma: A single surgeon's experience with 49 consecutive cases. World Neurosurg. 2015;84(6):1894-1902. DOI: 10.1016/j.wneu.2015.08.027

13. Canbay S, Hasturk AE, Basmaci M, et al. Management of thoracal and lumbar schwannomas using a unilateral approach without instability: An analysis of 15 cases. Asian Spine J. 2012;6(1):43-49. DOI: $10.4184 /$ asj.2012.6.1.43

14. Raynor RB, Pugh J, Shapiro I. Cervical facetectomy and its effect on spine strength. $J$ Neurosurg. 1985;63(2):278-282. DOI: 10.3171/jns.1985.63.2.0278

15. Nong L, Zhou D, Xu N, et al. Lamina replacement with titanium plate fixation improves spinal stability after total lumbar laminectomy. Comput Methods Biomech Biomed Engin. 2014;18:17. DOI: $10.1080 / 10255842.2014 .953491$

16. Yoganandan N, Maiman DJ, Pintar FA, et al. Biomechanical effects of laminectomy on thoracic spine stability. Neurosurgery. 1993;32(4):604-610. DOI: 10.1227/00006123-199304000-00017

17. Fehlings MG, Nater A, Zamorano JJ, et al. Risk factors for recurrence of surgically treated conventional spinal schwannomas: Analysis of 169 patients from a multicenter international database. Spine (Phila Pa 1976). 2016;41(5):390-398. DOI: 10.1097/BRS.0000000000001232

18. Fürstenberg $\mathrm{CH}$, Wiedenhöfer B, Gerner HJ, et al. The effect of early surgical treatment on recovery in patients with metastatic compression of the spinal cord. J Bone Joint Surg Br. 2009;91B(2):240-244. DOI: 10.1302/0301-620X.91B2.20894

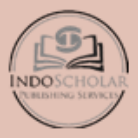
Articles published online under this model are officially publishedand can becited and quoted using the DOI as the reference source. Neurologico Spinale Medico Chirurgico has a policy that changes will not be made after publication of an article without following accepted procedures for making corrections to the scientific record. The entire contents of the Neurologico Spinale Medico Chirurgico are licensed under the $\mathrm{Creative}$ Commons Attribution 4.0 International License. You are free to share-copy \& redistribute the material in any medium or format, adapt - remix, transform, and build upon the material for any purpose, even commercially. Under the following terms, you must give appropriate credit, provide link to the license, and indicate if changes were made. You may do so in any reasonable manner, but not in any way that suggests the licensor endorses you or your use. 